\title{
polymers
}

ISSN 2073-4360

www.mdpi.com/journal/polymers

Article

\section{Preparation and Characterization of Organic-Inorganic Hybrid Hydrogel Electrolyte Using Alkaline Solution}

\author{
Masanobu Chiku, Shoji Tomita, Eiji Higuchi and Hiroshi Inoue * \\ Department of Applied Chemistry, Graduate School of Engineering, Osaka Prefecture University, \\ Sakai, Osaka 599-8531, Japan; E-Mails: chiku@chem.osakafu-u.ac.jp (M.C.); \\ s-tomita@chem.osakafu-u.ac.jp (S.T.); e-higuchi@chem.osakafu-u.ac.jp (E.H.) \\ * Author to whom correspondence should be addressed; E-Mail: inoue-h@chem.osakafu-u.ac.jp; \\ Tel.: +81-72-254-9283; Fax: +81-72-254-9283.
}

Received: 3 August 2011; in revised form: 29 August 2011 / Accepted: 21 September 2011 / Published: 26 September 2011

\begin{abstract}
Organic-inorganic hybrid hydrogel electrolytes were prepared by mixing hydrotalcite, cross-linked potassium poly(acrylate) and $6 \mathrm{M} \mathrm{KOH}$ solution. The organicinorganic hybrid hydrogel electrolytes had high ionic conductivity $\left(0.456-0.540 \mathrm{~S} \mathrm{~cm}^{-1}\right)$ at $30{ }^{\circ} \mathrm{C}$. Moreover, the mechanical strength of the hydrogel electrolytes was high enough to form a 2-3 $\mathrm{mm}$ thick freestanding membrane because of the reinforcement with hydrotalcite.
\end{abstract}

Keywords: hydrogel electrolyte; organic-inorganic hybrid; alkaline electrolyte

\section{Introduction}

Solid polymer electrolytes permitted safer, thinner and more flexible battery designs [1], but their ionic conductivity was insufficient to use in practice. Polymer gel electrolytes, which can stably hold considerable amount of electrolyte solution within polymer matrices, significantly improved ionic conductivity [2,3]. Thus lithium polymer secondary batteries with polymer gel electrolytes have actively been researched and developed. Meanwhile, alkaline secondary batteries such as nickel-metal hydride (Ni-MH) batteries are well-known as safe and environmentally friendly systems compared to 
lithium ion batteries and lithium polymer secondary batteries. Moreover, nickel-zinc and silver-zinc batteries are promising systems for their high energy density.

Several researchers have tried applying polymer electrolyte or gel electrolyte to alkaline secondary batteries [4-7]. Iwakura et al. firstly prepared a polymer hydrogel electrolyte (PHE) in which a $\mathrm{KOH}$ aqueous solution was held in a network structure of the cross-linked potassium poly(acrylate) (PAAK). They found that the PHE had liquidlike ionic conductivity, and applied it to all solid state Ni-MH batteries [8-10]. Inoue et al. developed a clay-based inorganic hydrogel electrolyte using hydrotalcite (HT) and $\mathrm{KOH}$ aqueous solution, and clarified that it had ionic conductivity comparable to the PHE [11]. However, these hydrogel electrolytes showed too low mechanical strength to form a freestanding membrane. In this work, we tried preparing organic-inorganic hybrid hydrogel electrolytes using PAAK, HT and $\mathrm{KOH}$ aqueous solution to realize not only high ionic conductivity but also high mechanical strength. Dispersing carbon black or silica as nanofiller is a popular strategy to reinforce rubber or organic polymer materials [12,13]. In addition, Croce et al. found that the nanocomposite polymer electrolytes using polyethylene oxide and $\mathrm{LiClO}_{4}$ containing $\mathrm{TiO}_{2}$ and $\mathrm{Al}_{2} \mathrm{O}_{3}$ nanoparticle had high ionic conductivity [14]. Therefore, it is reasonable to apply HT as nanofiller to reinforce the PHE.

\section{Experimental Section}

\subsection{Preparation of Organic-Inorganic Hybrid Hydrogel Electrolytes}

$\mathrm{KOH}$ and HT were purchased from Wako Pure Chemical Industries, Ltd. PAAK whose potassium content was $11 \mathrm{wt} \%$ was purchased from Sigma-Aldrich. Milli-Q (Millipore) water with resistivity of $18.2 \mathrm{M} \Omega \mathrm{cm}$ was used in this study. Three kinds of organic-inorganic hybrid hydrogel electrolytes were prepared according to the following procedure: $0.8,1.6$ or $2.4 \mathrm{~g}$ of HT was added to $10 \mathrm{~mL}$ of $6 \mathrm{M} \mathrm{KOH}$ aqueous solution. After being stirred for $5 \mathrm{~min}, 1 \mathrm{~g}$ of PAAK was added to the resultant suspension, followed by stirring for a few minutes and standing for 3 days, resulting in homogeneous milky hydrogel. After that, air bubbles in the gel were removed under a vacuum. All procedures were performed at room temperature. Each hydrogel electrolyte is called hybrid hydrogel $(0.8)$, hybrid hydrogel(1.6) and hybrid hydrogel(2.4), respectively.

\subsection{Electrochemical Measurements}

Electrochemical measurements were carried out using a potentiostat (SI1287 and SI1260; Solartron Analytical) with a three-electrode configuration cell. $\mathrm{An} \mathrm{Hg} / \mathrm{HgO}$ electrode was used as the reference electrode. Two Pt plates with apparent surface area of $1 \mathrm{~cm}^{2}$ were used as the counter and working electrodes, respectively. Ionic conductivity of $6 \mathrm{M} \mathrm{KOH}$ solution and three hydrogels was evaluated by ac impedance measurement at a frequency of $100 \mathrm{kHz}$ with the perturbation of $5 \mathrm{mV}$. A handmade two-electrode conductivity cell consisted of two Pt black-coated Pt electrodes with apparent surface area of $1 \mathrm{~cm}^{2}$ was used. 


\subsection{X-Ray Diffraction Analysis}

X-ray diffraction (XRD) spectra of HT and hybrid hydrogel electrolytes were measured by using an $\mathrm{X}$-ray diffractometer (XRD-6100; Shimadzu) equipped with a $\mathrm{Cu} K \alpha$ source $(\lambda=0.1541 \mathrm{~nm}, 50 \mathrm{kV}$, $30 \mathrm{~mA})$.

\section{Results and Discussion}

Figure 1 shows pictures of the hybrid hydrogel(1.6) and its freestanding membrane. The hybrid hydrogel(1.6) showed white color and tolerance to alkali. The hybrid hydrogel(1.6) kept its shape for hours when it was put on a flat surface (Figure 1(a,b)). in addition, it was possible to form a freestanding membrane with a thickness of 2-3 mm (Figure 1(c,d)). These strongly suggest the hydrogel electrolyte has high mechanical strength. The mechanical strength of the hydrogel electrolyte using only PAAK or HT was too low to form a self-standing membrane. Conventional polymer-clay composites containing aggregated nanolayer tactoids ordinarily improve rigidity, but they often sacrifice elongation and toughness [15]. However, the present hybrid hydrogel electrolytes keep both viscosity and elasticity. Thus, HT is homogeneously dispersed in PAAK matrix and HT works well as nanofiller to reinforce the PHE. Moreover, it should be noted that the hybrid hydrogel electrolytes are prepared just by mixing organic polymer, clay and $\mathrm{KOH}$ aqueous solution for only a few minutes. In this way, the hybrid hydrogel electrolytes are readily and quickly prepared.

Figure 1. Photographic images of hybrid hydrogel(1.6) and its freestanding membrane. (a) Top and (b) side views of the hybrid hydrogel(1.6); (c) front and (d) side views of the freestanding membrane.

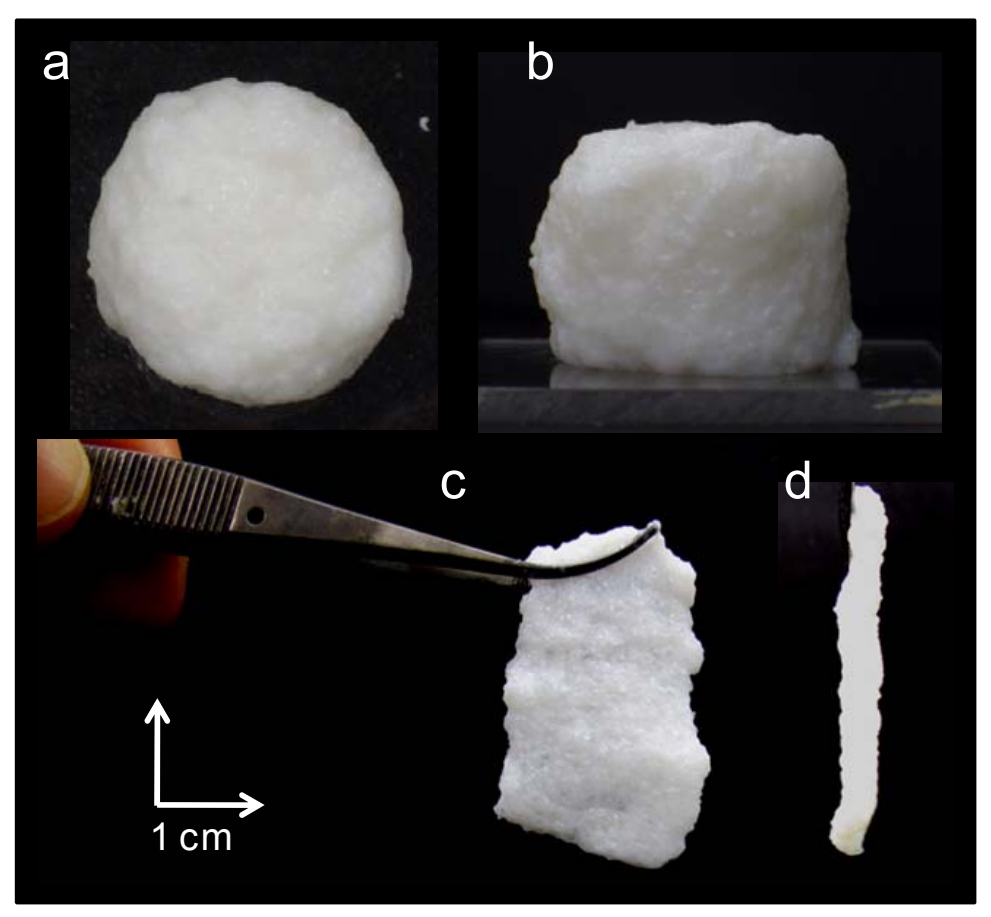

Figure 2 shows XRD spectra of the hybrid hydrogel(1.6) and pristine HT. The hybrid hydrogel(1.6) had diffraction peaks similar to the pristine HT. The lattice distance of the pristine HT was evaluated 
as $0.78 \mathrm{~nm}$ from the (003) peak and that of HT in the hybrid hydrogel(1.6) was $0.77 \mathrm{~nm}$. In the case of the inorganic hydrogel electrolyte with only HT, lattice distance was increased to $0.81 \mathrm{~nm}$ because carbonate was replaced with $\mathrm{OH}^{-}$upon ion exchange or the degree of hydration increased [11]. In the present case, it is deduced that the hybrid hydrogel(1.6) has lower degree of hydration than the inorganic hydrogel electrolyte. Another is less replacement of carbonate in the interlayer of HT lamellae with $\mathrm{OH}^{-}$ion. According to the Scherrer's equation, the crystallite size of HT was evaluated as $26.7 \mathrm{~nm}$ for the pristine HT and $24.6 \mathrm{~nm}$ for the hybrid hydrogel(1.6). Therefore, the number of HT lamellae is estimated to be 35 for the pristine HT and 33 for the hybrid hydrogel(1.6), indicating that most of the HT lamellae were not delaminated in the hybrid hydrogel.

Figure 2. X-ray diffraction (XRD) patterns of hybrid hydrogel(1.6) and pristine hydrotalcite (HT).

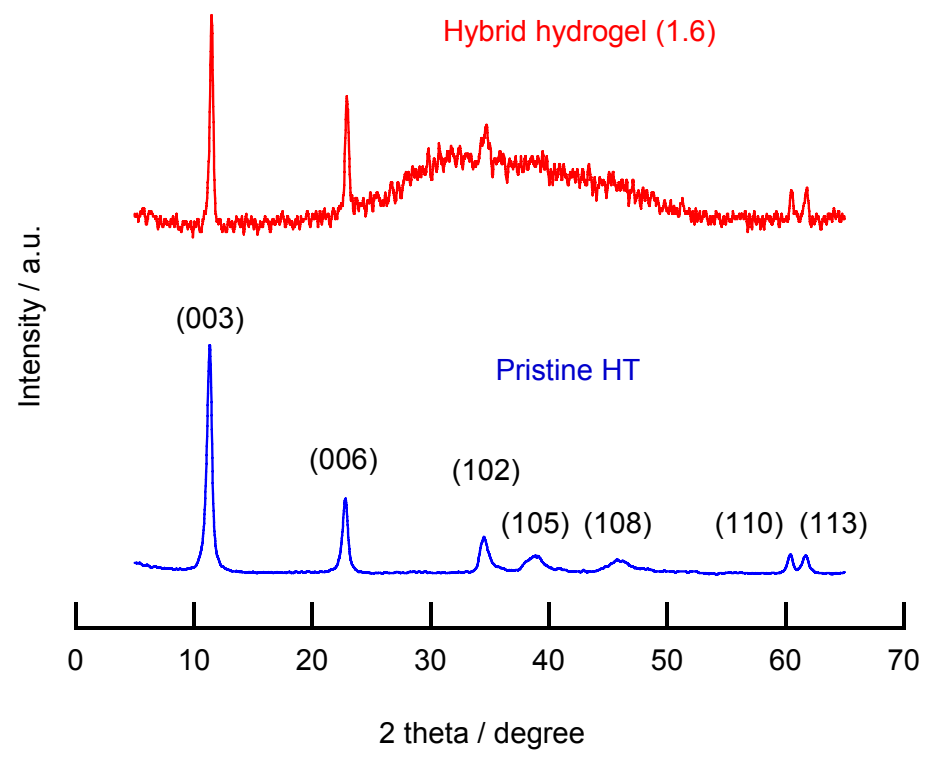

Figure 3 shows temperature dependence of ionic conductivity for $6 \mathrm{M} \mathrm{KOH}$ aqueous solution and three hybrid hydrogel electrolytes. Arrhenius equation is given as follows:

$$
\ln (\kappa)=\ln \sigma_{0}+\frac{\Delta E_{0}}{R} \cdot \frac{1}{T}
$$

where $\kappa$ is ionic conductivity, $R$ is the gas constant, $\sigma_{0}$ is frequency factor, $\Delta E_{0}$ is activation energy and $T$ is absolute temperature. In each case, logarithm of ionic conductivity in the range of 0 to $70{ }^{\circ} \mathrm{C}$ showed linear dependence on the reciprocal of absolute temperature. This suggests that the Arrhenius-type ionic conduction mechanism holds in the hybrid hydrogel electrolytes in the range of 0 to $70{ }^{\circ} \mathrm{C}$. Table 1 shows $\kappa$ at $30^{\circ} \mathrm{C}, \Delta E_{0}$ and $\ln \sigma_{0}$ values for three hybrid hydrogel electrolytes. The $\kappa$ at $30{ }^{\circ} \mathrm{C}, \Delta E_{0}$ and $\ln \sigma_{0}$ values for the $6 \mathrm{M} \mathrm{KOH}$ aqueous solution were $0.691 \mathrm{~S} \mathrm{~cm}^{-1}, 13.4 \mathrm{~kJ} \mathrm{~mol}^{-1}$ and 4.89, respectively. For the hybrid hydrogel electrolytes, ionic conductivity decreased with increasing HT content, while activation energy was independent of HT content, suggesting that the mobility of $\mathrm{OH}^{-}$ion did not change with $\mathrm{HT}$ content. On the other hand, $\ln \sigma_{0}$ gradually decreased with increasing HT content. This can be ascribed to the decrease of free $\mathrm{OH}^{-}$ions because carbonate anions adsorbing electrostatically on the HT particle surface with positive charges were replaced with $\mathrm{OH}^{-}$ions. 
Figure 3. Arrhenius plots of $6 \mathrm{M} \mathrm{KOH}$ solution and three hybrid hydrogel electrolytes.

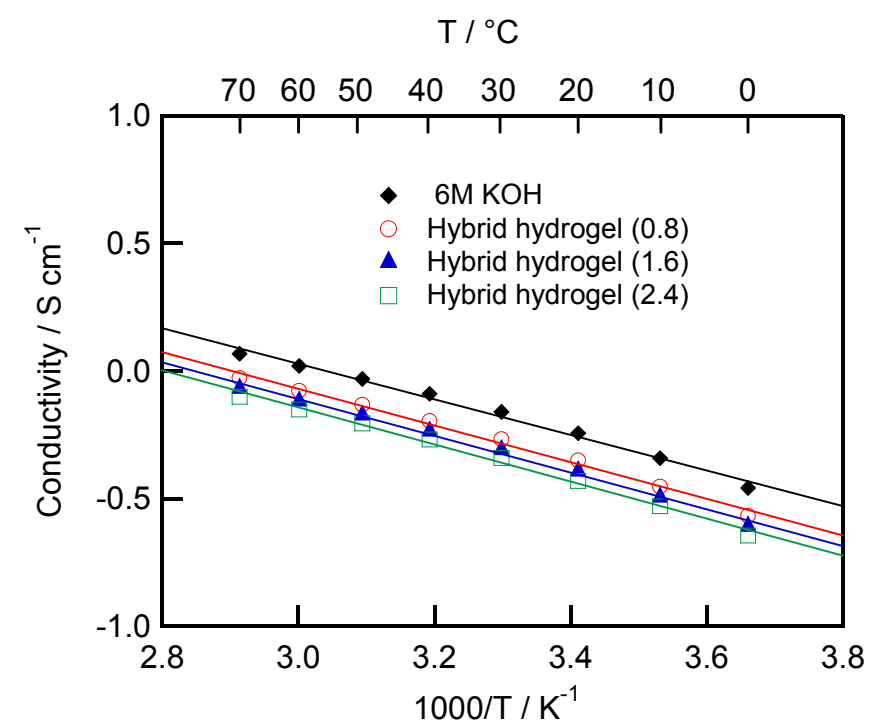

Table 1. Ionic conductivity $(\kappa)$, activation energy for ionic conductivity $\left(\Delta E_{0}\right)$ and logarithm of frequency factor $\left(\ln \sigma_{0}\right)$ for three hybrid hydrogel electrolytes.

\begin{tabular}{lccc}
\hline & Hybrid hydrogel(0.8) & Hybrid hydrogel(1.6) & Hybrid hydrogel(2.4) \\
\hline$\kappa$ at $30^{\circ} \mathrm{C} / \mathrm{S} \mathrm{cm}^{-1}$ & 0.540 & 0.491 & 0.456 \\
$\Delta E_{0} / \mathrm{kJ} \mathrm{mol}^{-1}$ & 13.8 & 13.8 & 13.9 \\
$\ln \left(\sigma_{0} / \mathrm{S} \mathrm{cm}^{-1}\right)$ & 4.80 & 4.72 & 4.69 \\
\hline
\end{tabular}

Figure 4 shows cyclic voltammograms $(\mathrm{CVs})$ of a Pt working electrode in $6 \mathrm{M} \mathrm{KOH}$ aqueous solution and three hybrid hydrogel electrolytes. Typical oxidation and reduction peaks due to the formation of $\mathrm{Pt}$ oxide and its reduction and hydrogen adsorption and desorption were observed in the $\mathrm{CV}$ in each hybrid hydrogel electrolyte. However, there was no significant current for any other reactions in each voltammogram. Therefore we can say the electrochemical window of Pt in the hybrid hydrogel electrolytes is comparable to that in $6 \mathrm{M} \mathrm{KOH}$ aqueous solution, which clearly indicates the hybrid hydrogel electrolytes are promising solid electrolytes for alkaline batteries, capacitors and fuel cells compatible with $6 \mathrm{M} \mathrm{KOH}$ aqueous solution.

Figure 4. Cyclic voltammograms of a Pt electrode in three hybrid hydrogel electrolytes at $30{ }^{\circ} \mathrm{C}$. Scan rate: $100 \mathrm{mV} \mathrm{s}^{-1}$.

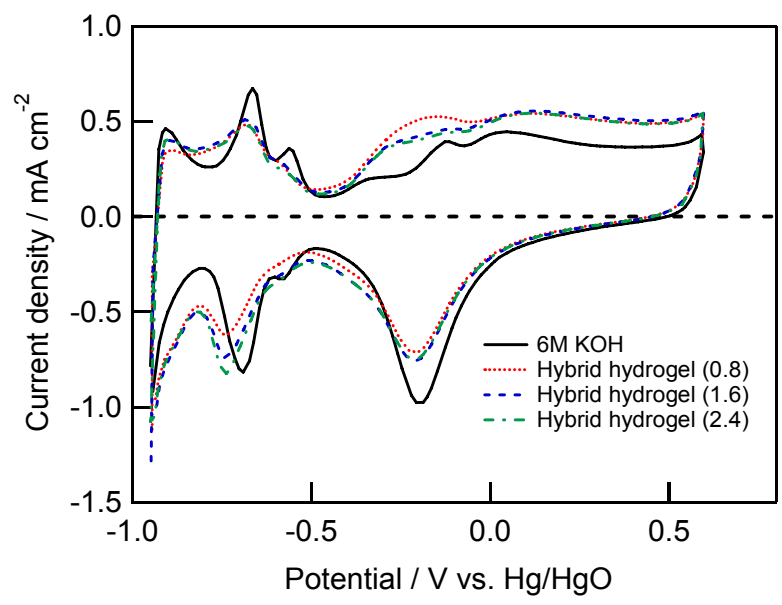




\section{Conclusions}

The mechanical strength of the hybrid hydrogel electrolytes containing both PAAK and HT is high enough to form freestanding membranes. Their ionic conductivity is $0.456-0.540 \mathrm{~S} \mathrm{~cm}^{-1}$ at $30{ }^{\circ} \mathrm{C}$ and their potential window is comparable to that in $\mathrm{KOH}$ solution. Moreover, the hybrid hydrogel electrolytes can be prepared just by mixing the HT, PAAK and $\mathrm{KOH}$ solution without polymerization and thermal treatment. The hybrid hydrogel electrolytes have potential for safe, economical and environmentally friendly energy applications like batteries, capacitors and fuel cells.

\section{References}

1. Meyer, W.H. Polymer electrolytes for lithium-ion batteries. Adv. Mater. 1998, 10, 439-448.

2. Abraham, K.M.; Alamgir, M. $\mathrm{Li}^{+}$-Conductive solid polymer electrolytes with liquid-like conductivity. J. Electrochem. Soc. 1990, 137, 1657-1658.

3. Sotomura, T.; Tatsuma, T.; Oyama, N. An organosulfur polymer cathode with a high current capability for rechargeable batteries. J. Electrochem. Soc. 1996, 143, 3152-3157.

4. Fauvarque, J.F.; Guinot, S.; Bouzir, N.; Salmon, E.; Penneau, J.F. Alkaline Poly(ethylene oxide) solid polymer electrolytes. Application to nickel secondary batteries. Electrochim. Acta 1995, 40, 2449-2453.

5. Vassal, N.; Salmon, E.; Fauvarque, J.F. Nickel/metal hydride secondary batteries using an alkaline solid polymer electrolyte. J. Electrochem. Soc. 1999, 146, 20-26.

6. Lewandowski, A.; Skorupska, K.; Malinska, J. Novel poly(vinyl alcohol)-KOH- $\mathrm{H}_{2} \mathrm{O}$ alkaline polymer electrolyte. Solid State Ion. 2000, 133, 265-271.

7. Kumar, G.G.; Sampath, S. Electrochemical and spectroscopic investigations of a gel polymer electrolyte of Poly(Methylmethacrylate) and zinc triflate. Solid State Ion. 2005, 176, 773-780.

8. Iwakura, C.; Furukawa, N.; Ohnishi, T.; Sakamoto, K.; Nohara, S.; Inoue, H. Nickel/metal hydride cells using an alkaline polymer gel electrolyte based on potassium salt of crosslinked of Poly (Acrylic Acid). Electrochemistry (Tokyo, Jpn.) 2001, 69, 659-663.

9. Iwakura, C.; Ikoma, K.; Nohara, S.; Furukawa, N.; Inoue, H. Charge-discharge and capacity retention characteristics of new type $\mathrm{Ni} / \mathrm{MH}$ batteries using polymer hydrogel electrolyte. J. Electrochem. Soc. 2003, 150, A1623-A1627.

10. Iwakura, C.; Nohara, S.; Furukawa, N.; Inoue, H. The possible use of polymer gel electrolytes in nickel/metal hydride battery. Solid State Ion. 2002, 148, 487-492.

11. Inoue, H.; Inaba, Y.; Okuda, S.; Higuchi, E.; Nohara, S. Inorganic hydrogel electrolyte with liquidlike ionic conductivity. Electrochem. Solid-State Lett. 2009, 12, A58-A60.

12. Ueno, K.; Hata, K.; Katakabe, T.; Kondoh, M.; Watanabe, M. Nanocomposite ion gels based on silica nanoparticles and an ionic liquid: Ionic transport, viscoelastic properties, and microstructure. J. Phys. Chem. B 2008, 112, 9013-9019.

13. Haraguchi, K.; Takehisa, T. Nanocomposite hydrogels: A unique organic-inorganic network structure with extraordinary mechanical, optical, and swelling/de-swelling properties. Adv. Mater. 2002, 14, 1120-1124. 
14. Croce, F.; Appetechi, G.B.; Persi, L.; Scrosati, B. Nanocomposite polymer electrolytes for lithium batteries. Nature 1998, 394, 456-459.

15. LeBaron, P.C.; Wang, Z.; Pinnavaia, T.J. Polymer-layered silicate nanocomposites: An overview. Appl. Clay Sci. 1999, 15, 11-29.

(C) 2011 by the authors; licensee MDPI, Basel, Switzerland. This article is an open access article distributed under the terms and conditions of the Creative Commons Attribution license (http://creativecommons.org/licenses/by/3.0/). 